\title{
Interlayer exciton valleytronics in bilayer heterostructures interfaced with a phase gradient metasurface
}

Cite as: Appl. Phys. Lett. 117, 121101 (2020); https://doi.org/10.1063/5.0015087

Submitted: 25 May 2020 . Accepted: 02 September 2020 . Published Online: 21 September 2020

Mandar Sohoni (D), Pankaj K. Jha (D), Muralidhar Nalabothula, and Anshuman Kumar (D)

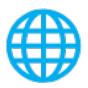

\section{Lock-in Amplifiers up to $600 \mathrm{MHz}$}
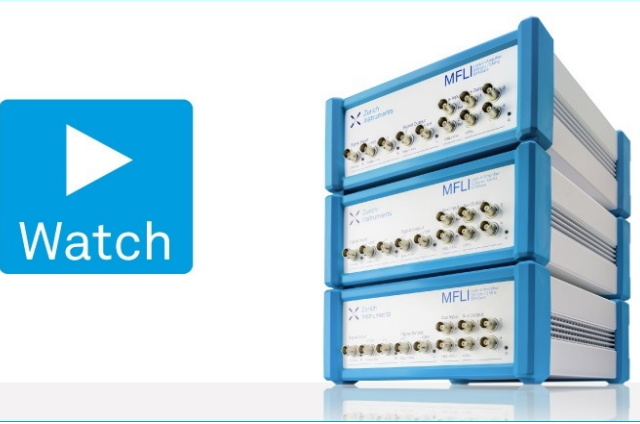

Appl. Phys. Lett. 117, 121101 (2020); https://doi.org/10.1063/5.0015087

117, 121101 


\title{
Interlayer exciton valleytronics in bilayer heterostructures interfaced with a phase gradient metasurface
}

\author{
Cite as: Appl. Phys. Lett. 117, 121101 (2020); doi: 10.1063/5.0015087 \\ Submitted: 25 May 2020 - Accepted: 2 September 2020 • \\ Published Online: 21 September 2020

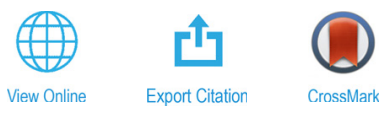

Mandar Sohoni, ${ }^{7}$ (D) Pankaj K. Jha, ${ }^{2}$ (D) Muralidhar Nalabothula, ${ }^{3}$ and Anshuman Kumar ${ }^{1, a)}$ (D)

\begin{abstract}
AFFILIATIONS
${ }^{7}$ Physics Department, Indian Institute of Technology Bombay, Mumbai 400076, India

${ }^{2}$ Thomas J. Watson Laboratories of Applied Physics, California Institute of Technology, Pasadena, California 91125, USA

${ }^{3}$ Physics and Materials Science Research Unit, University of Luxembourg, 162a avenue de la Faïencerie, L-1511 Luxembourg, Luxembourg

${ }^{a)}$ Author to whom correspondence should be addressed: anshuman.kumar@iitb.ac.in
\end{abstract}

\begin{abstract}
Interlayer excitons in bilayer heterostructures of two-dimensional semiconductors have opened a new platform for valley physics and optoelectronic applications owing to several unique properties. Here, we theoretically demonstrate the coherent manipulation of the interlayer excitonic valley degree of freedom in a transition metal dichalcogenide heterobilayer interfaced with a phase gradient metasurface. We harness the peculiar out-of-plane component of polarization of interlayer excitons in conjunction with the anisotropy of the vacuum of the electromagnetic field enabled by the metasurface to induce coherent coupling between mutually orthogonal interlayer excitonic optical selection rule. This coupling leads to a much larger domain within the valley psuedo-spin Bloch sphere being accessible in these heterostructures. Our proposed route to coherent manipulation of interlayer excitons in bilayer heterostructures with structured interfaces such as a metasurface may pave the way for engineering an array of interacting quantum emitters in Moiré heterostructures for chip-scale quantum information science.
\end{abstract}

Published under license by AIP Publishing. https://doi.org/10.1063/5.0015087

In recent years, valleytronics-the technology to manipulate the electronic valley degree of freedom in two dimensional gapped Dirac systems, which possess pairs of degenerate band extrema or valleyshas received enormous attention for quantum information processing applications. ${ }^{1,2}$ Few layer transition metal dichalcogenides (TMDCs) are one class of semiconductors that have shown great promise for such technologies. In TMDC heterostructures, such as $\mathrm{MoX}_{2} / \mathrm{WX}_{2}$, there exists a type II band alignment, which results in the interlayer exciton being the most energetically favorable. ${ }^{3,4}$ In addition, their heterostructures are rather easily formed without the constraints of epitaxial matching, and they can be integrated with other photonic platforms. ${ }^{5,6}$ There have been several proposals to use TMDC heterostructures to develop optoelectronic devices, similar to those in the field of spintronics. ${ }^{7,8}$ Interlayer excitons, due to their large binding energy $(\sim 0.25 \mathrm{eV}){ }^{9}$, are stable even at room temperature; thus, such heterostructures have the potential to host valleytronics devices without the need for cryogenic systems. State initialization and readout of these pseudo-spins are optically possible due to the valleys' coupling to orthogonal polarizations of light. ${ }^{1}$ It turns out that, as we show below, such heterostructures can not only host in-plane circularly polarized excitons (similar to monolayers), but also circularly polarized excitons that have their quantization axes tilted. The kinds of excitons formed in these heterostructures depend on the interlayer translation (lateral shift between unit cells) between the top and bottom layer. ${ }^{10}$

In this Letter, we introduce a hybrid nano-photonic system comprising of bilayer $\mathrm{MoX}_{2} / \mathrm{WX}_{2}$ heterostructure interfaced with a phase gradient metasurface to investigate the coherent dynamics of interlayer excitons in the heterostructure. It is interesting to study such phenomena in heterostructures for the following reasons. First, interlayer excitons, due to the spatial separation of the electron-hole pair, have much smaller intervalley scattering rates ${ }^{11}$ as compared to their intralayer counterparts. Second, Moiré superlattices can generate an array of such interlayer excitons; thus, they have the potential to host interacting quantum emitter arrays which have enormous potential for information processing. ${ }^{12-15}$ Finally, as we will show below, due to the form of the valley exciton coupling in intralayer excitons, circularly 
polarized dipoles will only have a real intervalley coupling constant for diagonal Green's tensors which limits the valley pseudo-spin to only reside in the $x z$ plane of the Bloch sphere. Interlayer excitons on the other hand, while retaining in-plane circularly polarized dipoles, also have "non-trivial" tilted circularly polarized dipoles. These non-trivial dipoles have complex coupling constants, thus resulting in a larger accessible region of the Bloch sphere.

As with any scheme for quantum control, one must be able to generate coherence between these two excitons. Broadly, there are two approaches to generate such coherence between the valleys-one that involves an external coherent electromagnetic pump ${ }^{16-18}$ and another, spontaneously, using an anisotropic vacuum generation. ${ }^{19,20}$ The latter approach is relatively less explored in the context of TMDCs and is the subject of this work. To generate the anisotropic vacuum, we used a custom designed array of nano-antennas, i.e., a metasurface. Metasurfaces, a special kind of planar metamaterial, allow user defined electromagnetic waveform responses with control over properties such as amplitude, phase, and polarization ${ }^{21-23}$ with an ultra-thin spatial footprint. Figure 1 shows a schematic of the proposed TMDC heterostructure placed above the metasurface. Here, we designed polarization sensitive nano-antennas (see the supplementary material) such that the metasurface induces local in-plane anisotropic vacuum of the electromagnetic field which enables coupling between two orthogonal valleys of the $\mathrm{MoSe}_{2} / \mathrm{WSe}_{2}$ heterostructure. Similar to a classical focusing device like a lens/mirror, our metasurface can also tailor the vacuum

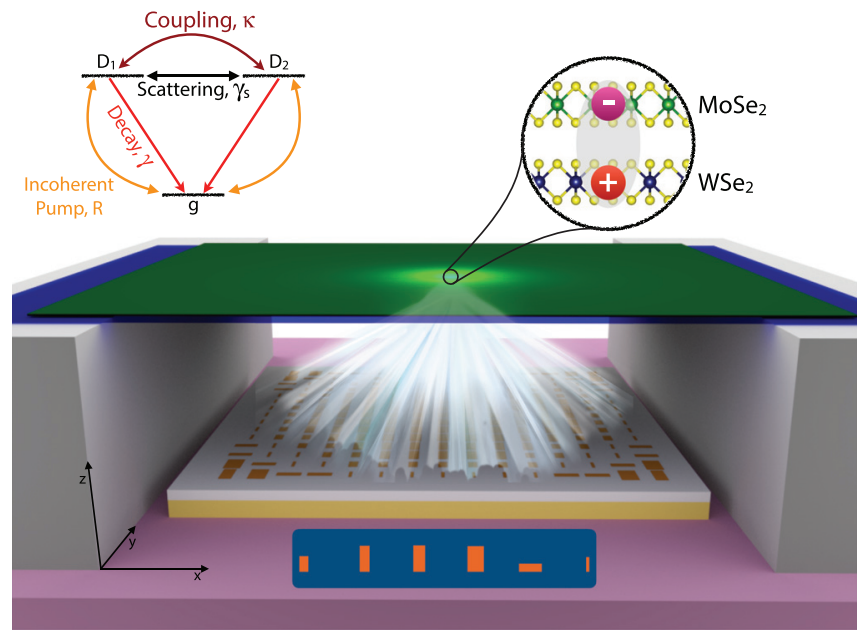

FIG. 1. A schematic of a hybrid nano-photonic system comprising of TMDC heterostructure interfaced with a phase gradient metasurface. The zoomed-in inset on the right depicts the interlayer exciton. In an isotropic electromagnetic vacuum, such as the case with free space, orthogonal interlayer excitons $( \pm K \pm K$ for $R$ type stacking and $\pm K \mp K$ for $H$ type stacking) will not interact with each other due to optical selection rule. Our metasurface, which consists of judiciously designed gold nanoantenna with a gold back-reflector and a dielectric spacer layer (alumina) sandwiched between them, creates a local in-plane anisotropic environment which allows a finite non-zero coupling between mutually orthogonal interlayer excitons. The inset on the left pictorially depicts the metasurface enabled interaction between the orthogonal excitons in a V-scheme. The lower inset shows six gold nanoantennas with dimensions $(90,160),(101,267),(120,271),(166,263),(233,82)$, $(30,150)$ to cover the phase $0-2 \pi$ with a step of $\pi / 3$. Here, all dimensions are in $\mathrm{nm}$. The thickness of all nano-antennas is $30 \mathrm{~nm}$. The refractive index of gold and alumina was taken from reference. ${ }^{4}$ of the electromagnetic field within the spatial range of $\sim \lambda / 2$ about the focal point located at a distance of few tens of wavelengths from the metasurface. We modeled the interlayer exciton as a dipole whose position should be sufficiently far from the metasurface such that it "sees" the metasurface rather than one antenna, i.e., the exciton dynamics is governed by metasurface. However, the farther the excitonic-dipole, the larger is the size of the metasurface required.

Valley excitons $(+K$ or $-K)$ in monolayer TMDCs are circularly polarized in the plane of the monolayer. ${ }^{24}$ Such systems can be modelled as a V-level scheme with the two transitions being orthogonal, but degenerate. ${ }^{17,19,20}$ Valley excitons in TMDC bilayer heterostructures, however, have dipole moments that can couple to right circularly, left circularly, and linearly $(z)$ polarized light. ${ }^{25}$ In general, the interlayer valley exciton dipole moments can be written as

$$
\begin{aligned}
& \mathbf{D}_{1}=a_{+}^{+K} \mathbf{e}_{+}+a_{-}^{+K} \mathbf{e}_{-}+a_{z}^{+K} \mathbf{e}_{z}, \\
& \mathbf{D}_{2}=a_{+}^{-K} \mathbf{e}_{+}+a_{-}^{-K} \mathbf{e}_{-}+a_{z}^{-K} \mathbf{e}_{z},
\end{aligned}
$$

where $\mathbf{D}_{1}=\mathbf{D}_{+K \pm K}$ and $\mathbf{D}_{2}=\mathbf{D}_{-K \mp K}$, depending on the stacking $(\mathrm{R} / \mathrm{H}), \mathbf{e}_{ \pm}=(\hat{x}+i \hat{y}) / \sqrt{2}$, and $\mathbf{e}_{z}=\hat{z}$. The coefficients $a_{ \pm, z}^{ \pm K}$ are dependent on the interlayer translation $\mathbf{r}_{0}$ (see the supplementary material). In this work, we are interested in those configurations where the dipoles in the two valleys are orthogonal, that is, when $\hat{\mathbf{D}}_{2}^{*} \cdot \hat{\mathbf{D}}_{1}=0$, because these dipoles can then be viewed as a pseudospin. Most of the values of $r_{0}$ for which $\hat{\mathbf{D}}_{2}^{*} \cdot \hat{\mathbf{D}}_{1}=0$ are the high symmetry points $\left(r_{0}=0, \frac{1}{3}\right.$, or $\left.\frac{2}{3}\right)$ where the dipoles are in-plane circularly polarized, similar to the monolayer case. However, there are a few non-trivial zeros where the orthogonal dipoles are not in-plane circularly polarized, but have all three components in the laboratory frame. It turns out that these non-trivial zeros are in fact circularly polarized dipoles with a quantization axis that is tilted. The quantization axis $\bar{n}$ of these non-trivial zeros is given by

$$
\bar{n}_{1(2)}=\operatorname{Re}\left(\hat{\mathbf{D}}_{1(2)}\right) \times \operatorname{Im}\left(\hat{\mathbf{D}}_{1(2)}\right),
$$

and since $\hat{\mathbf{D}}_{2}^{*} \cdot \hat{\mathbf{D}}_{1}=0 \Rightarrow \operatorname{Re}\left(\hat{\mathbf{D}}_{1(2)}\right) \cdot \operatorname{Im}\left(\hat{\mathbf{D}}_{1(2)}\right)=0$, the non-trivial dipoles are circularly polarized in the plane defined $\bar{n}_{1(2)}{ }^{26}$ (note that $\bar{n}_{1}=-\bar{n}_{2}$, see the supplementary material). Even though commensurate TMDC bilayer heterostructures with most of the interlayer translation values, $r_{0}$, do not exist naturally, they can be found in incommensurate heterostructures with small twist angles (moiré superlattices). ${ }^{10,27}$ They are found on length scales larger than the monolayer lattice constants, but smaller than the moire supercell lattice vectors. In these regions, the atom placement is indistinguishable from that of a commensurate stacking with interlayer translation. Figure 2(a) shows the variation of $\left|\hat{\mathbf{D}}_{2}^{*} \cdot \hat{\mathbf{D}}_{1}\right|$ with $r_{0}$ for singlet excitons in the example of a commensurate $\mathrm{H}$ type stacking, which is the focus of this work. One should note that there are multiple interlayer translations for which the excitonic dipoles are orthogonal; however, only two of them correspond to tilted circularly polarized excitonic dipoles (one has been marked with a cross). Our formalism can be easily extended to other types of stacking, which are further discussed in the supplementary material.

In an isotropic medium, the coupling between any two orthogonal transitions is forbidden due to optical selection rule. One proposal to work around this was to create an anisotropic vacuum of an electromagnetic field that would result in a non-zero coupling between orthogonal transitions. ${ }^{28}$ In-plane anisotropic vacuum can be generated by using metasurfaces, ${ }^{29}$ or by using anisotropic polaritonic 
a)
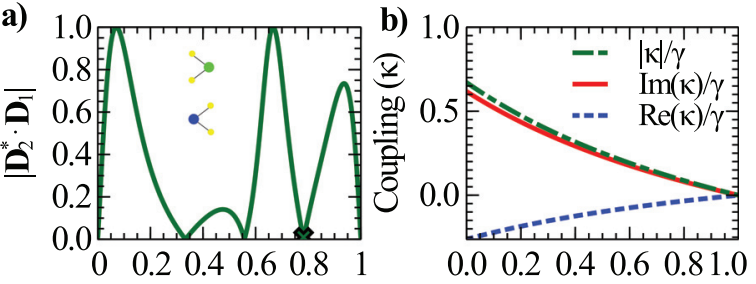

Interlayer Translation $\left(\mathrm{r}_{0}\right)$

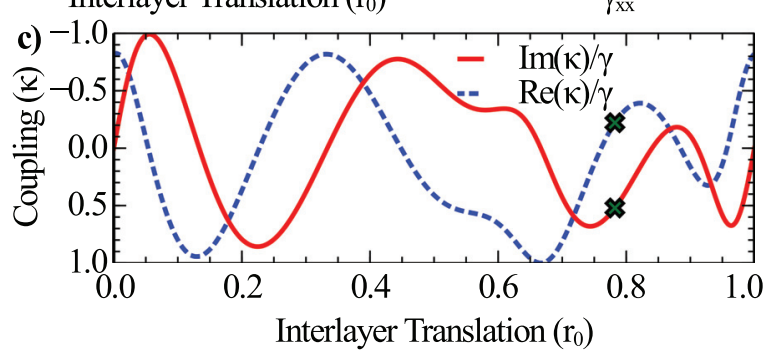

FIG. 2. Singlet exciton in a commensurate $\mathrm{H}$ type $\mathrm{MoSe}_{2} / \mathrm{WSe}_{2}$ heterostructure: (a) the absolute value of the dot product between $\hat{\mathbf{D}}_{2}$ and $\hat{\mathbf{D}}_{1}$ as a function of the interlayer translation $r_{0}$. The inset depicts an $\mathrm{H}$ type stacking order. (b) Variation of $\operatorname{Re}(\kappa) / \gamma, \operatorname{Im}(\kappa) / \gamma$, and $|\kappa| / \gamma$ with $\gamma_{x x}$ for the dipole marked by the cross in (b). We have taken $\gamma_{y y}$ and $\gamma_{z z}$ to be 1. (c) Variation of $\operatorname{Re}(\kappa) / \gamma$ and $\operatorname{Im}(\kappa) / \gamma$ with the interlayer translation $r_{0}$ when $\gamma_{x x}=0.1$ and $\gamma_{y y}=\gamma_{z z}=1$ in accordance with the values expected from our metasurface design. The cross marks the values of the real and imaginary part of the coupling at the non-trivial zero marked in (a). The non-trivial zero that we chose to design the metasurface for is marked with a cross.

materials. ${ }^{19}$ In general, the coupling rate between the two orthogonal dipoles is given by ${ }^{28}$

$$
\tilde{\kappa}_{21}=\frac{2 \omega_{0}^{2}}{\hbar \epsilon_{0} c^{2}} \mathbf{D}_{2}^{*} \cdot \operatorname{Im}[\overleftrightarrow{\mathbf{G}}] \cdot \mathbf{D}_{1},
$$

where $\overrightarrow{\mathbf{G}}$ is the total Green's tensor at the position of the dipoles. In general, the Green's tensor $\overleftrightarrow{\mathbf{G}}$ is a complex-valued function where the imaginary part, Im $[\overleftrightarrow{\mathbf{G}}]$, leads to coupling [Eq. (4)] and the real part, $\operatorname{Re}[\overrightarrow{\mathbf{G}}]$, leads to frequency shift. A shift in resonance frequency is detrimental to quantum interference. ${ }^{28}$ We designed our metasurface such that $\operatorname{Re}[\overleftrightarrow{\mathbf{G}}]$ is negligible. The normalized coupling rate, $\tilde{\kappa}_{21} / \gamma_{0}$, for inplane circular dipoles evaluates to $\left(\gamma_{x x}-\gamma_{y y}\right) / 2$, where $\gamma_{j j}$ is the normalized decay rate for a dipole with $j$ polarization, i.e., $\gamma_{x x(y y)}$ $\propto \mathbf{x}(\mathbf{y})^{*} \cdot \operatorname{Im}[\overrightarrow{\mathbf{G}}] \cdot \mathbf{x}(\mathbf{y})$. Here, $\gamma_{0}$ is the free space decay rate ${ }^{30}$ (see Sec. IV of the supplementary material). Since $\kappa$ has been normalized, it is independent of the dipole strength (which is small for interlayer excitons), and we expect to see metasurface governed dynamics on timescales of $\gamma_{0}^{-1}$. One must note that a non-unity quantum efficiency, $\eta$, will change Eq. $(4)^{31}$ (see supplementary material Sec. VI for details about the effect of $\eta$ ). For subsequent analysis, we have taken $\eta=1$, in light of low temperature experiments that have measured a rather high value of $\eta .^{30,32}$ In an anisotropic vacuum, where $\gamma_{x x} \neq \gamma_{y y}$, the coupling will be a finite non-zero value. It should be noted that this coupling constant is always real. For the case of interlayer exciton, however, a completely different form of coupling constant emerges, namely, $\kappa=\tilde{\kappa}_{21} / \gamma_{0}=\kappa_{x x}+\kappa_{y y}+\kappa_{z z}$, where

$$
\kappa_{x x, y y}= \pm \frac{\gamma_{x x, y y}\left(a_{+}^{+K} \pm a_{-}^{+K}\right)^{2}}{2\left(\left|a_{+}^{+K}\right|^{2}+\left|a_{-}^{+K}\right|^{2}+\left|a_{z}^{+K}\right|^{2}\right)},
$$

$$
\kappa_{z z}=\frac{\gamma_{z z}\left(a_{z}^{+K}\right)^{2}}{\left|a_{+}^{+K}\right|^{2}+\left|a_{-}^{+K}\right|^{2}+\left|a_{z}^{+K}\right|^{2}} .
$$

If $\gamma_{x x}=\gamma_{y y}=\gamma_{z z}$, the coupling constant will be proportional to the dot product of the two dipoles and will be 0 for orthogonal transitions. A table of the values of $\kappa / \gamma$ for different stackings and types ( $\mathrm{R}$ and $\mathrm{H}$; singlet and triplet) for the non-trivial zeros can be found in the supplementary material.

To show the dependence of the coupling on the interlayer translation, in Fig. 2(c) we plot the variation of the real and imaginary parts of $\kappa / \gamma$ for the singlet exciton in a commensurate $\mathrm{H}$-type $\mathrm{MoSe}_{2} / \mathrm{WSe}_{2}$ heterostructure. We consider a metasurface which is optimized such that $\gamma_{x x}=0.1$, and $\gamma_{y y}=\gamma_{z z}=1$ for each value of the interlayer translation. The values of $\gamma_{i i}$ chosen are in accordance with what was expected from our metasurface. Figure 2(b) shows the variation of the real and imaginary parts of $\kappa / \gamma$ with $\gamma_{x x}\left(\gamma_{y y, z z}=1\right)$ for the exciton marked in Fig. 2(a), which confirms that our chosen values of the $\gamma_{i i}$ 's are indeed close to optimal for observing the largest coupling rate. It must be noted, however, that in the case of other types of stacking, the optimal values of the corresponding decay rates might differ. In this work, we use a nano-antenna based metasurface to generate an anisotropy between the $x$ and $y$ directions. Our metasurface is designed to act as a normal mirror for the $y$-dipole and a spherical mirror for the $x$-dipole. It is worth mentioning that phase of the scattered field, reflected back at the location of interlayer excitonic-dipole, can be engineered to interfere constructively or destructively with the dipole field. Without loss of generality, we chose our metasurface phase profile which enables destructive interference for the $x$-dipole. This inplane polarization selectivity is a unique feature of our phase gradient metasurface. In this work, we consider the excitonic-dipole to be located at a distance of $d=10 \lambda(\lambda \approx 874 \mathrm{~nm}$ is the chosen exciton's wavelength) from the metasurface which serves two purposes: First, the dipole is distant enough to "see" the metasurface rather than the nearest meta-atom, and second, its dynamics can be controlled by our moderately sized metasurface of dimensions $\sim 100 \lambda$. Our gold nanoantenna based metasurface with average reflection (intensity) $>86 \%$ gives us $\gamma_{x x} \approx 0.1$ and $\gamma_{y y}=1$, which is quite close to the optimal value as highlighted in Fig. 2(c).

To measure the coherence between two in-plane circularly polarized dipoles, a quantity known as the degree of linear polarization (DoLP) has been proposed. ${ }^{33}$ This is the normalized Stokes' parameter $\mathrm{S}_{1} \cdot{ }^{34}$ For systems such as ours, this metric only captures a part of the coherence in the system since it corresponds to a projective measurement in the $x z$ plane of the Bloch sphere. To capture the full coherence, we also need to consider the third Stokes' parameter, $S_{2}$. In steady state, the Stokes' parameters take the form (see the supplementary material for the rate equations)

$$
\begin{aligned}
& \frac{\mathrm{S}_{2}}{\mathrm{~S}_{0}}=\frac{i\left(\rho_{12}-\rho_{21}\right)}{\rho_{11}+\rho_{22}}=\frac{\kappa_{i}}{\beta}, \\
& \frac{\mathrm{S}_{1}}{\mathrm{~S}_{0}}=\frac{\rho_{12}+\rho_{21}}{\rho_{11}+\rho_{22}}=-\frac{\kappa_{r}}{\beta},
\end{aligned}
$$

where $\kappa_{r(i)}$ is the real (imaginary) part of $\kappa$ and $\beta=\gamma+R+\gamma_{s}$. Here, $\gamma$ is the spontaneous decay rate of the dipoles in the presence of the metasurface, $\gamma_{s}$ is the intervalley scattering rate, ${ }^{35}$ and $R$ is the incoherent pump rate. In order to calculate these experimentally measurable 
parameters, we solve for the density matrix of the system via the master equation as follows: ${ }^{36}$

$$
\begin{aligned}
\dot{\rho}= & i \sum_{n} \Delta \omega_{n}[|n\rangle\langle n|, \rho]+i \sum_{m \neq n} \delta_{n m}[|n\rangle\langle m|, \rho] \\
& +\sum_{n \neq m} \Gamma_{n m}\left(\rho_{m n}|g\rangle\langle g|-\frac{1}{2}\{|n\rangle\langle m|, \rho\}\right) \\
& +\sum_{n} \Gamma_{n n}\left(\rho_{n n}|g\rangle\langle g|-\frac{1}{2}\{|n\rangle\langle n|, \rho\}\right) \\
& +R \sum_{n}\left[\left(\rho_{g g}|n\rangle\left\langle n\left|+\rho_{n n}\right| g\right\rangle\langle g|\right)-\frac{1}{2}\{|n\rangle\langle n|, \rho\}\right] \\
& +\gamma_{s} \sum_{n \neq m}\left[\rho_{n n}|m\rangle\langle m|-\frac{1}{2}\{|n\rangle\langle n|, \rho\}\right],
\end{aligned}
$$

where $n, m=1,2 .|g\rangle$ denotes the ground state, $\Gamma_{21}=\kappa=\Gamma_{12}^{*}$ is the coupling constant and captures the two photon (virtual) process between the two orthogonal dipoles, $\Gamma_{n n}$ is the spontaneous decay rate of the dipole, $\delta_{n m}$ is the resonant dipole-dipole interaction, and $\Delta \omega_{n}$ accounts for the lamb-shift. For our system, both $\delta_{n m}$ and $\Delta \omega_{n}$ are two orders of magnitude smaller than $\Gamma_{n m}$ and, hence, have been neglected. Figure 3(a) shows the evolution of the Stokes parameters on the Poincaré sphere with bi-directional incoherent pumping for both $\hat{\mathbf{D}}_{1}$ and $\hat{\mathbf{D}}_{2}$ with the initial state prepared in $\hat{\mathbf{D}}_{1}$ (see the left inset of Fig. 1). For our heterostructure, the system evolves outside the $S_{1}-S_{3}$ plane. It must be noted that for the monolayer TMDC in the absence of any external field (optical or magnetic), the polarization trajectory is confined to the $S_{1}-S_{3}$ plane only. The interlayer exciton in heterobilayer system, therefore, offers unprecedented spontaneous access to the rest of the Poincare sphere. For comparison, we have included a trajectory for a system where there is no metasurface $(\kappa=0)$. In Fig. $3(\mathrm{~b})$, we show that with metasurfaces of different design, the phase $\phi=\tan ^{-1}\left(S_{2} / S_{1}\right)$ of the projection of the polarization state on the (a)

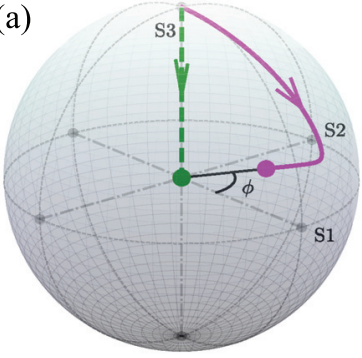

(b)

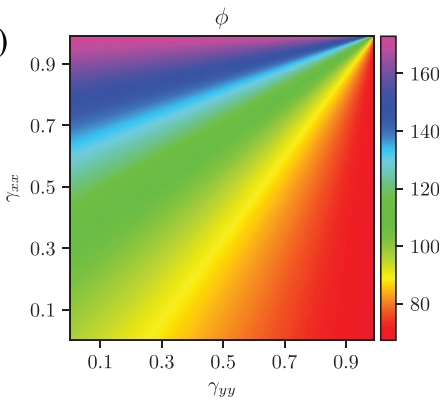

FIG. 3. Stokes parameters with bi-directional incoherent pumping for both $\hat{\mathbf{D}}_{1}$ and $\hat{\mathbf{D}}_{2}$ for the H-type singlet exciton marked by the cross in Fig. 2(a). (a) Temporal evolution of the Stokes' parameters represented on the Poincare sphere. Magenta is for our heterostructure in the presence of the metasurface and green is in the absence of a metasurface (an isotropic vacuum). We have taken the system initially to be in one valley. The cirlces at the end of the trajectories denote the steady state and are on the $S_{1}-S_{2}$ plane. One should note that the system in our heterostructure evolves outside the $S_{1}-S_{3}$ plane. (b) A two-dimensional plot of the phase, $\phi=\operatorname{atan}\left(\mathrm{S}_{2} / \mathrm{S}_{1}\right)$, marked in (a) for different values of $\gamma_{x x}$ and $\gamma_{y y}\left(\gamma_{z z}=1\right)$. The parameters used in the simulation are $R=0.001 \gamma_{0}, \gamma=0.639 \gamma_{0}$, $\kappa=(-0.142+i 0.333) \gamma_{0}, \gamma_{s}=0.1 \gamma_{0}$. equatorial plane can be tuned for different values of $\gamma_{x x}$ and $\gamma_{y y}$ $\left(\gamma_{z z}=1\right)$. One should note that $\gamma_{x x / y y}$ is tunable via the geometrical design of the metasurface and enables accessing different states on the Poincaré sphere. While Fig. 3(b) only shows the range of $\phi$ when we have suppressed $\gamma_{x x, y y}$, one can access the entire range of $\phi$ if the decay rates can also be enhanced. ${ }^{20}$ (see supplementary material Fig. S2.)

To summarize, we have shown a valleytronics application of spontaneous coherence between interlayer excitons in commensurate TMDC heterostructures. We have found the existence of tilted circular orthogonal dipoles in $\mathrm{R}$ and $\mathrm{H}$ commensurate stackings of $\mathrm{MoSe}_{2} / \mathrm{WSe}_{2}$ heterostructures which could potentially be used to observe non-inverse dynamics (different temporal evolutions for different initial states of the emitter). ${ }^{37}$ We have analyzed the coupling between these kinds of dipoles in the presence of an anisotropic vacuum created by a nano-antenna based metasurface. The couplings are complex, which allow the system to evolve outside the $x z$ plane of the Bloch sphere-providing access to a very large part of the Bloch sphere, overcoming a fundamental limitation of all previous works related to monolayer TMDCs in an anisotropic vacuum. Our analysis of spontaneous coherence for interlayer excitons may pave the way for engineering an array of interacting quantum emitters in Moiré heterostructures. ${ }^{38}$ In addition to the valley degree of freedom, bilayer heterostructures also have a layer degree of freedom, ${ }^{8}$ which can be electrically tuned. ${ }^{39}$ Other avenues to be explored are systems where the $\operatorname{Re}[\overrightarrow{\mathbf{G}}]$ is large and plasmonic systems where $\gamma_{x x / y y}$ can be tuned using gate voltages. ${ }^{19}$ Heterostructures interfaced with such systems could potentially offer many coherent operations before decoherence of the state.

See the supplementary material contains the details about the dipole moments, the metasurface design, orthogonal dipoles in different stacking orders, and the effect of non-unity quantum efficiency.

A. Kumar acknowledges funding support from the Department of Science and Technology via Grant Nos. SB/S2/RJN110/2017, DST/NM/NS-2018/49, and ECR/2018/001485.

\section{DATA AVAILABILITY}

The data that support the findings of this study are available within the article (and its supplementary material).

\section{REFERENCES}

IJ. R. Schaibley, H. Yu, G. Clark, P. Rivera, J. S. Ross, K. L. Seyler, W. Yao, and X. Xu, Nat. Rev. Mater. 1, 1 (2016).

${ }^{2}$ F. Langer, C. P. Schmid, S. Schlauderer, M. Gmitra, J. Fabian, P. Nagler, C. Schüller, T. Korn, P. G. Hawkins, J. T. Steiner, U. Huttner, S. W. Koch, M. Kira, and R. Huber, Nature 557, 76 (2018).

${ }^{3}$ P. Rivera, H. Yu, K. L. Seyler, N. P. Wilson, W. Yao, and X. Xu, Nat. Nanotechnol. 13, 1004 (2018).

${ }^{4}$ O. Karni, E. Barré, S. C. Lau, R. Gillen, E. Y. Ma, B. Kim, K. Watanabe, T. Taniguchi, J. Maultzsch, K. Barmak, R. H. Page, and T. F. Heinz, Phys. Rev. Lett. 123, 247402 (2019).

${ }^{5}$ Y. Liu, Y. Huang, and X. Duan, Nature 567, 323 (2019).

${ }^{6}$ K. S. Novoselov, A. Mishchenko, A. Carvalho, and A. H. C. Neto, Science $\mathbf{3 5 3}$, aac9439 (2016).

${ }^{7}$ T. Cao, G. Wang, W. Han, H. Ye, C. Zhu, J. Shi, Q. Niu, P. Tan, E. Wang, B. Liu, and J. Feng, Nat. Commun. 3, 887 (2012). 
${ }^{8}$ Z. Gong, G.-B. Liu, H. Yu, D. Xiao, X. Cui, X. Xu, and W. Yao, Nat. Commun. 4, 2053 (2013).

${ }^{9}$ M.-H. Chiu, M.-Y. Li, W. Zhang, W.-T. Hsu, W.-H. Chang, M. Terrones, H. Terrones, and L.-J. Li, ACS Nano 8, 9649 (2014).

${ }^{10}$ C. Zhang, C.-P. Chuu, X. Ren, M.-Y. Li, L.-J. Li, C. Jin, M.-Y. Chou, and C.-K. Shih, Sci. Adv. 3, el601459 (2017).

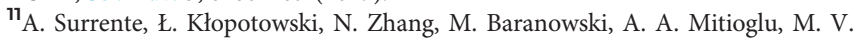
Ballottin, P. C. Christianen, D. Dumcenco, Y.-C. Kung, D. K. Maude, A. Kis, and P. Plochocka, Nano Lett. 18, 3994 (2018).

${ }^{12}$ H. Yu, G.-B. Liu, J. Tang, X. Xu, and W. Yao, Sci. Adv. 3, e1701696 (2017).

${ }^{13}$ K. L. Seyler, P. Rivera, H. Yu, N. P. Wilson, E. L. Ray, D. G. Mandrus, J. Yan, W. Yao, and X. Xu, Nature 567, 66 (2019).

${ }^{14}$ C. Jin, E. C. Regan, D. Wang, M. I. B. Utama, C.-S. Yang, J. Cain, Y. Qin, Y. Shen, Z. Zheng, K. Watanabe, T. Taniguchi, S. Tongay, A. Zettl, and F. Wang, Nat. Phys. 15, 1140 (2019).

${ }^{15} \mathrm{~F}$. Wu, T. Lovorn, and A. H. MacDonald, Phys. Rev. B 97, 035306 (2018).

${ }^{16}$ A. M. Jones, H. Yu, N. J. Ghimire, S. Wu, G. Aivazian, J. S. Ross, B. Zhao, J. Yan, D. G. Mandrus, D. Xiao, W. Yao, and X. Xu, Nat. Nanotechnol. 8, 634 (2013).

${ }^{17}$ K. Hao, G. Moody, F. Wu, C. K. Dass, L. Xu, C.-H. Chen, L. Sun, M.-Y. Li, L.-J. Li, A. H. MacDonald, and X. Li, Nat. Phys. 12, 677 (2016).

${ }^{18}$ B. Zhu, H. Zeng, J. Dai, Z. Gong, and X. Cui, Proc. Natl. Acad. Sci. 111, 11606 (2014).

${ }^{19}$ M. Nalabothula, P. K. Jha, T. Low, and A. Kumar, Phys. Rev. B 102, 045416 (2020).

${ }^{20} \mathrm{P}$. K. Jha, N. Shitrit, X. Ren, Y. Wang, and X. Zhang, Phys. Rev. Lett. 121, 116102 (2018).

${ }^{21}$ S. Jahani and Z. Jacob, Nat. Nanotechnol. 11, 23 (2016).

${ }^{22}$ A. V. Kildishev, A. Boltasseva, and V. M. Shalaev, Science 339, 1232009 (2013).
${ }^{23}$ N. Yu and F. Capasso, Nat. Mater. 13, 139 (2014).

${ }^{24}$ D. Xiao, G.-B. Liu, W. Feng, X. Xu, and W. Yao, Phys. Rev. Lett. 108, 196802 (2012).

${ }^{25}$ H. Yu, G.-B. Liu, and W. Yao, 2D Mater. 5, 035021 (2018).

${ }^{26}$ I. V. Lindell, Methods for Electromagnetic Field Analysis (IEEE Press, 1992).

${ }^{27}$ Y. Wang, Z. Wang, W. Yao, G.-B. Liu, and H. Yu, Phys. Rev. B 95, 115429 (2017).

${ }^{28}$ G. S. Agarwal, Phys. Rev. Lett. 84, 5500 (2000).

${ }^{29}$ P. K. Jha, X. Ni, C. Wu, Y. Wang, and X. Zhang, Phys. Rev. Lett. 115, 025501 (2015).

${ }^{30}$ P. Rivera, J. R. Schaibley, A. M. Jones, J. S. Ross, S. Wu, G. Aivazian, P. Klement, K. Seyler, G. Clark, N. J. Ghimire et al., Nat. Commun. 6, 1 (2015).

${ }^{31}$ L. Novotny and B. Hecht, Principles of Nano-Optics (Cambridge University Press, 2012).

${ }^{32}$ L. A. Jauregui, A. Y. Joe, K. Pistunova, D. S. Wild, A. A. High, Y. Zhou, G. Scuri, K. De Greve, A. Sushko, C.-H. Yu et al., Science 366, 870 (2019).

${ }^{33}$ L. Qiu, C. Chakraborty, S. Dhara, and A. N. Vamivakas, Nat. Commun. 10, 1 (2019).

${ }^{34}$ J. Altepeter, E. Jeffrey, and P. Kwiat, Advances in Atomic, Molecular, and Optical Physics (Elsevier, 2005), pp. 105-159.

${ }^{35}$ P. Rivera, K. L. Seyler, H. Yu, J. R. Schaibley, J. Yan, D. G. Mandrus, W. Yao, and X. Xu, Science 351, 688 (2016).

${ }^{36}$ S. Hughes and G. S. Agarwal, Phys. Rev. Lett. 118, 063601 (2017).

${ }^{37}$ D. Kornovan, M. Petrov, and I. Iorsh, Phys. Rev. A 100, 033840 (2019).

${ }^{38}$ Y. Tang, J. Gu, S. Liu, K. Watanabe, T. Taniguchi, J. Hone, K. F. Mak, and J. Shan, arXiv:2002.10067 (2020).

${ }^{39}$ S. Gao, L. Yang, and C. D. Spataru, Nano Lett. 17, 7809 (2017).

${ }^{40}$ E. D. Palik, Handbook of Optical Constants of Solids (Academic Press, 1998), Vol. 3. 